\title{
Solving the Linear Oscillatory Problem without Damping with Random Loading Condition Using the Decomposition Method
}

\author{
Amnah S. Al-Juhani*, Aleh A. Al-Shammari \\ Faculty of Science, Tabuk University, Tabuk, KSA \\ Email: *aalgohani@ut.edu.sa, ‘xxwhitelinnetxx@hotmail, L0L0.10@hotmail.com
}

How to cite this paper: Al-Juhani, A.S. and Al-Shammari, A.A. (2019) Solving the Linear Oscillatory Problem without Damping with Random Loading Condition Using the Decomposition Method. Journal of Applied Mathematics and Physics, 7, 527-535. https://doi.org/10.4236/jamp.2019.73038

Received: April 2, 2018

Accepted: March 10, 2019

Published: March 13, 2019

Copyright $\odot 2019$ by authors and Scientific Research Publishing Inc. This work is licensed under the Creative Commons Attribution International License (CC BY 4.0).

http://creativecommons.org/licenses/by/4.0/

\section{c) (i) Open Access}

\begin{abstract}
In this paper we study the solution of random linear oscillatory equation $\ddot{x}+w^{2} x=F(t ; \omega)$ without damping and with random leading condition using the method. Finally, the time evolution of the mean, variance and standard deviation has been plotted for a range of values of the natural frequency $w$.
\end{abstract}

\section{Keywords}

Linear Stochastic Differential Equations, Adomian Decompositions, Linear Oscillatory, Mathematica

\section{Introduction}

The Adomian decomposition technique was firstly introduced by Adomian in 1975. This technique can be used to solve differential, integral, algebraic and many other equations (linear or nonlinear) [1]-[12]. The method is based on a suggestion by Adomian G. that the solution can be decomposed into components. In the coming sections we will see that the Adomian decomposition method is also very convenient computationally and offers some significant advantages [13]-[20]. The Adomian decomposition method is not a perturbation procedure, so no assumption concerning the size of randomness is necessary, where each term from the decomposed solution depends only on the preceding terms. A little work in the convergence of the procedure had been done [21] [22] [23] [24] [25].

\section{Problem Formulation}

In this paper, we focus on solving the following Solving the linear oscillatory problem 


$$
\begin{gathered}
\ddot{x}+w^{2} x=F(t ; \omega) \\
F(t ; \omega)=e(t)[1+\varepsilon n(t ; \omega)]
\end{gathered}
$$

under stochastic excitation $F(t ; \omega)$ with the deterministic initial conditions

$$
x(0)=x_{0}, \quad \dot{x}(0)=\dot{x}_{0}
$$

where

$w$. frequency of oscillation,

$\varepsilon$ : deterministic nonlinearity scale,

$\omega \in(\Omega, \sigma, P):$ a triple probability space with $\Omega$ as the sample space, where $\sigma$ is a $\sigma$-algebra on event in $\Omega$ and $P$ is a probability measure, and $n(t ; \omega)$ is a white noise with the following properties:

$$
\begin{gathered}
E n(t ; \omega)=0 \\
E n\left(t_{1} ; \omega\right) \cdot n\left(t_{2} ; \omega\right)=\operatorname{cov}\left[n\left(t_{1}\right), n\left(t_{2}\right)\right]=\delta\left(t_{1}-t_{2}\right)
\end{gathered}
$$

By obtaining the P.d.f. of $x(t)$, the average and variance of the solution process in terms of $\mathrm{t}$ : time, the general solution is

$$
x(t)=x_{0} \cos w t+\frac{\dot{x}_{0}}{w} \sin w t+\frac{1}{\omega} \int_{0}^{t} \sin w(t-s) F(s ; q) \mathrm{d} s
$$

The ensemble average is given by

$$
\begin{aligned}
E x(t) & =\mu_{x(t)}=x_{0} \cos w t+\frac{\dot{x}_{0}}{w} \sin w t+\frac{1}{w} \int_{0}^{t} \sin w(t-s) E F(s ; q) \mathrm{d} s \\
& =x_{0} \cos w t+\frac{\dot{x}_{0}}{w} \sin w t+\frac{1}{\omega} \int_{0}^{t} \sin w(t-s) e(s) \mathrm{d} s
\end{aligned}
$$

The covariance takes the form

$$
\begin{aligned}
\operatorname{cov}\left(x\left(t_{1}\right), x\left(t_{2}\right)\right) & =E\left(x\left(t_{1}\right)-\mu_{x\left(t_{1}\right)}\right) \cdot\left(x\left(t_{2}\right)-\mu_{x\left(t_{2}\right)}\right) \\
& =\frac{\varepsilon^{2}}{w^{2}} \int_{0}^{t_{1}} \sin w\left(t_{1}-s\right) \sin w\left(t_{2}-s\right) e^{2}(s) \mathrm{d} s
\end{aligned}
$$

The variance is

$$
\sigma_{x}^{2}(t)=\frac{\varepsilon^{2}}{w^{2}} \int_{0}^{t} \sin ^{2} w(t-s) e^{2}(s) \mathrm{d} s
$$

Due to linearity and the deterministic properties of $x_{0}, \dot{x}_{0}$ and the frequency $w$ we obtain a Gaussian solution process:

$$
f_{x(t)}=\frac{1}{\sigma_{x(t)} \sqrt{2 \pi}} \mathrm{e}^{-\frac{1}{2}\left(\frac{x(t)-\mu_{x(t)}}{\sigma_{x(t)}}\right)^{2}}
$$

where $\mu_{x(t)}=x_{0} \cos w t+\frac{\dot{x}_{0}}{\omega} \sin w t+\frac{1}{\omega} \int_{0}^{t} \sin w(t-s) e(s) \mathrm{d} s$. 


$$
\sigma_{x(t)}^{2}=\frac{\varepsilon^{2}}{w^{2}} \int_{0}^{t} \sin ^{2} w(t-s) e^{2}(s) \mathrm{d} s
$$

Equation (9) represents a closed form solution of problem (1) with random loading condition.

\section{The Adomian Decomposition Method}

\section{Case-study:}

Let us consider

$$
F(t ; \omega)=\mathrm{e}^{-t}+\varepsilon n(t ; \omega)
$$

In the Adomian decomposition method, differential operators are decomposed. Thus Equation (1) is rewritten in the following form:

$$
(L+R) x=F(t ; q)
$$

where:

$$
L=\frac{\mathrm{d}^{2}}{\mathrm{~d} t^{2}} \text { and } R=\omega^{2}
$$

Hence,

$$
L x=F(t ; q)-R x
$$

Solving for $x$ we obtain

$$
x=L^{-1} F(t ; q)-L^{-1} R x+\phi(t)
$$

where $\phi(t)$ is the solution of $L x=0$

$$
L x=0 \Rightarrow \frac{\mathrm{d}^{2} x}{\mathrm{~d} t^{2}}=0 \Rightarrow x=a t+c
$$

Subject to the initial conditions:

$$
\phi(t)=x_{0}+\dot{x}_{0} t
$$

Thus, the solution of equation takes the form:

$$
x=x_{0}+\dot{x}_{0} t+\int_{0}^{t} \int_{0}^{t} F(t ; q) \mathrm{d} t \mathrm{~d} t-w^{2} \int_{0}^{t} \int_{0}^{t} x(t) \mathrm{d} t \mathrm{~d} t
$$

We now assume that the solution can be written in the following form:

$$
x(t)=x^{(0)}(t)+x^{(1)}(t)+\cdots=\sum_{i=0}^{\infty} x^{(i)}(t)
$$

Substituting (17) in (16) we obtain:

$$
\sum_{i=0}^{\infty} x^{(i)}=x_{0}+\dot{x}_{0} t+\int_{0}^{t} \int_{0}^{t} F(t ; q) \mathrm{d} t \mathrm{~d} t-\omega^{2} \sum_{i=0}^{\infty} \int_{0}^{t} \int_{0}^{t} x^{(i)}(t) \mathrm{d} t \mathrm{~d} t
$$

By matching the boundaries, we obtain:

$$
x^{(0)}(t)=x_{0}+\dot{x}_{0} t+\int_{0}^{t} \int_{0}^{t} F(t ; q) \mathrm{d} t \mathrm{~d} t
$$




$$
\begin{gathered}
x^{(1)}(t)=-w^{2} \int_{0}^{t} \int_{0}^{t} x^{(0)} \mathrm{d} t \mathrm{~d} t \\
x^{(2)}(t)=-w^{2} \int_{0}^{t} \int_{0}^{t} x^{(1)}(t) \mathrm{d} t \mathrm{~d} t
\end{gathered}
$$

And the nth term will be:

$$
x^{(n)}(t)=-w^{2} \int_{0}^{t} \int_{0}^{t} x^{(n-1)}(t) \mathrm{d} t \mathrm{~d} t, n \geq 1
$$

By applying this procedure to equation, we obtain:

$$
\begin{aligned}
& x^{(1)}(t)=-w^{2} x_{o} \frac{t^{2}}{2 !}-w^{2} \dot{x}_{0} \frac{t^{3}}{3 !}-w^{2} L^{-1} L^{-1} F(t ; q) \\
& x^{(2)}(t)=w^{4} x_{0} \frac{t^{4}}{4 !}+w^{4} \dot{x}_{0} \frac{t^{5}}{5 !}+w^{4} L^{-1} L^{-1} L^{-1} F(t ; q) \\
& x^{(3)}(t)=-w^{6} x_{0} \frac{t^{6}}{6 !}-w^{6} \dot{x}_{0} \frac{t^{7}}{7 !}-w^{6}\left(L^{-1}\right)^{4} F(t ; q) \\
& x^{(4)}(t)=w^{8} x_{0} \frac{t^{8}}{8 !}+w^{8} \dot{x}_{0} \frac{t^{9}}{9 !}+w^{8}\left(L^{-1}\right)^{5} F(t ; q)
\end{aligned}
$$

The nth term is:

$$
x^{(n)}(t)=w^{2 n} x_{0} \frac{t^{2 n}}{2 n !}+w^{2 n} \dot{x}_{0} \frac{t^{2 n+1}}{(2 n+1) !}+w^{2 n}\left(L^{-1}\right)^{n+1} F(t ; q)
$$

Thus,

$$
\begin{aligned}
& x(t)=x^{(0)}+x^{(1)}+x^{(2)}+\cdots \\
& =x_{0}\left[1-\frac{(w t)^{2}}{2 !}+\frac{(w t)^{4}}{4 !}+\cdots\right]+\frac{\dot{x}_{0}}{\omega}\left[(w t)-\frac{(w t)^{3}}{3 !}+\frac{(w t)^{5}}{5 !}+\cdots\right] \\
& +\frac{1}{w}\left[w L^{-1}-w^{3}\left(L^{-1}\right)^{2}+w^{5}\left(L^{-1}\right)^{3}-w^{7}\left(L^{-1}\right)^{4}+w^{9}\left(L^{-1}\right)^{5}+\cdots\right] F(t ; q) \\
& =x_{0} \cos \omega t+\frac{\dot{x}_{0}}{\omega} \sin \omega t+\frac{1}{\omega}\left[\omega L^{-1}-\omega^{2}\left(L^{-1}\right)^{2}\right] F(t ; q)
\end{aligned}
$$

where,

$$
\begin{gathered}
\int_{0}^{t} \cdots \int_{0}^{t} F(u) \mathrm{d} u^{n}=\int_{0}^{t} \frac{(t-u)^{n-1}}{(n-1) !} F(u) \mathrm{d} u \\
L^{-1} F(t ; q)=\int_{0}^{t} \int_{0}^{t} F(t ; q) \mathrm{d} t^{2}=\int_{0}^{t}(t-u) F(u ; q) \mathrm{d} u \\
L^{-1} L^{-1} F(t ; q)=\int_{0}^{t} \int_{0}^{t} \int_{0}^{t} \int_{0}^{t} F(t ; q) \mathrm{d} t^{4}=\int_{0}^{t} \frac{(t-u)^{3}}{3 !} F(u ; q) \mathrm{d} u \\
L^{-1} L^{-1} L^{-1} F(t ; q)=\int_{0}^{t} \int_{0}^{t} \int_{0}^{t} \int_{0}^{t} \int_{0}^{t} \int_{0}^{t} F(t ; q) \mathrm{d} t^{6}=\int_{0}^{t} \frac{(t-u)^{5}}{5 !} F(u ; q) \mathrm{d} u
\end{gathered}
$$




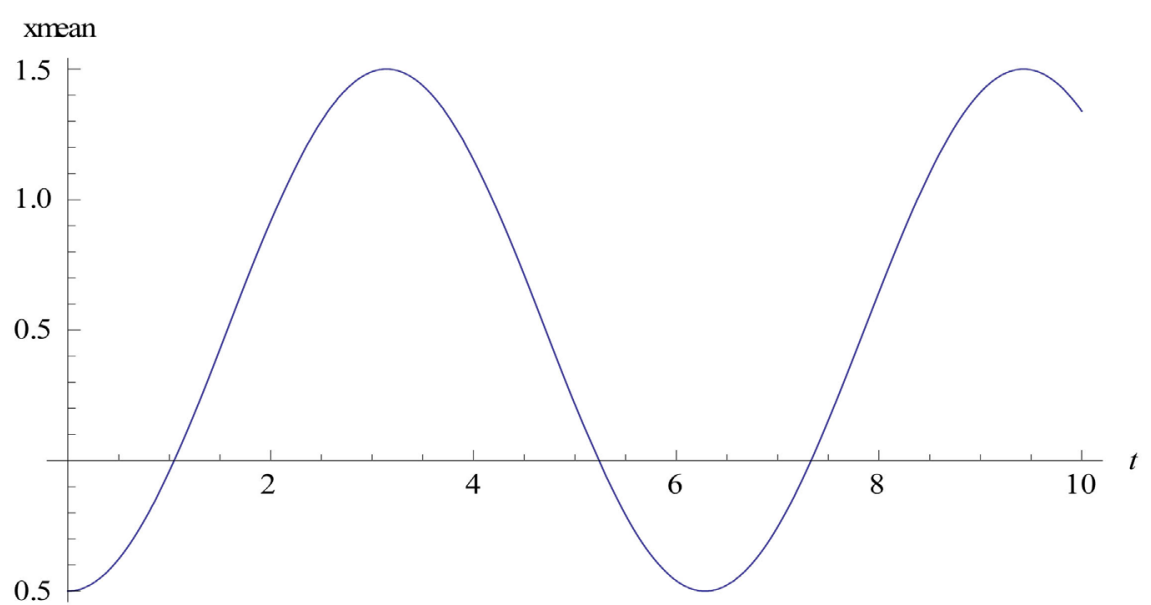

Figure 1. The mean of $x(t)$ at $\omega=1$.

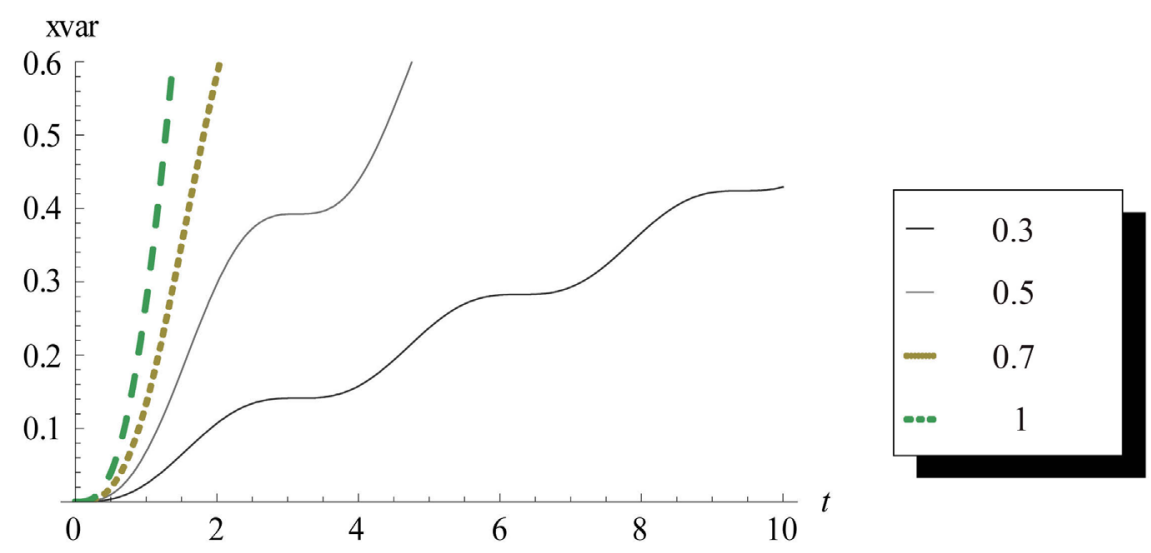

Figure 2. The variance of $x(t)$ at $\omega=1$.

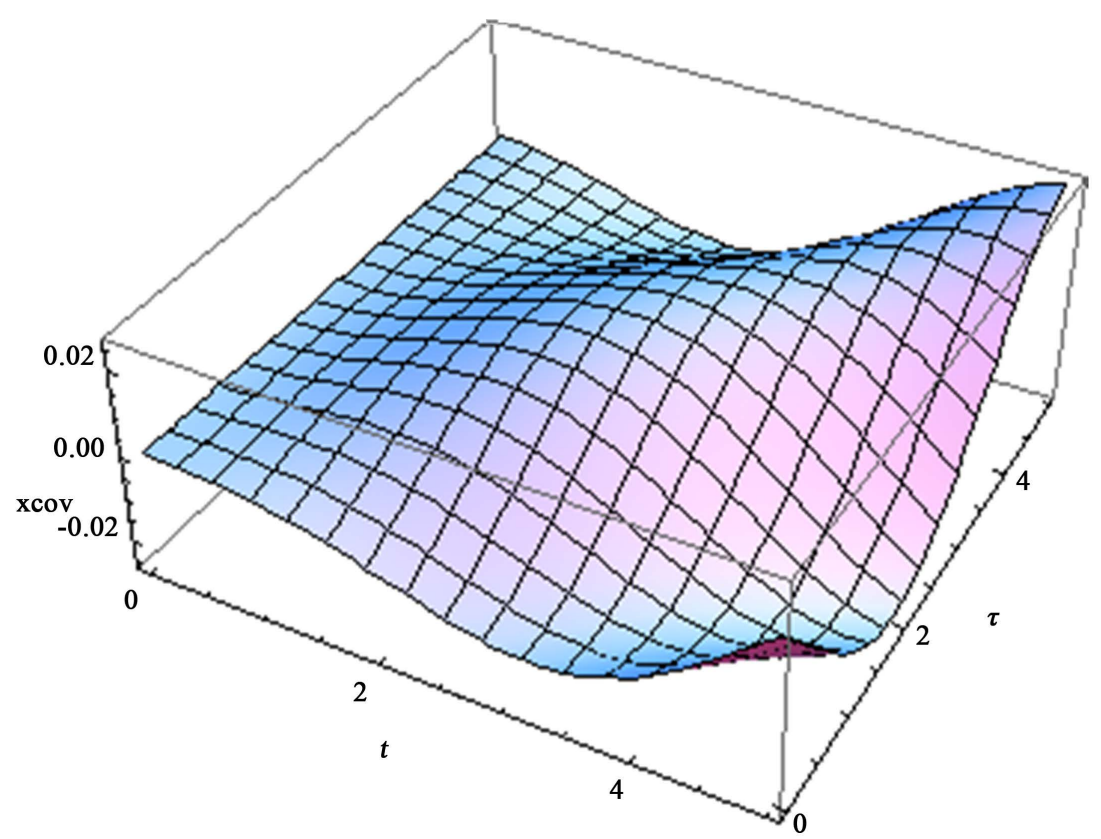

Figure 3. The covariance of $x(t)$ at $\varepsilon=0.1, \omega=1$. 


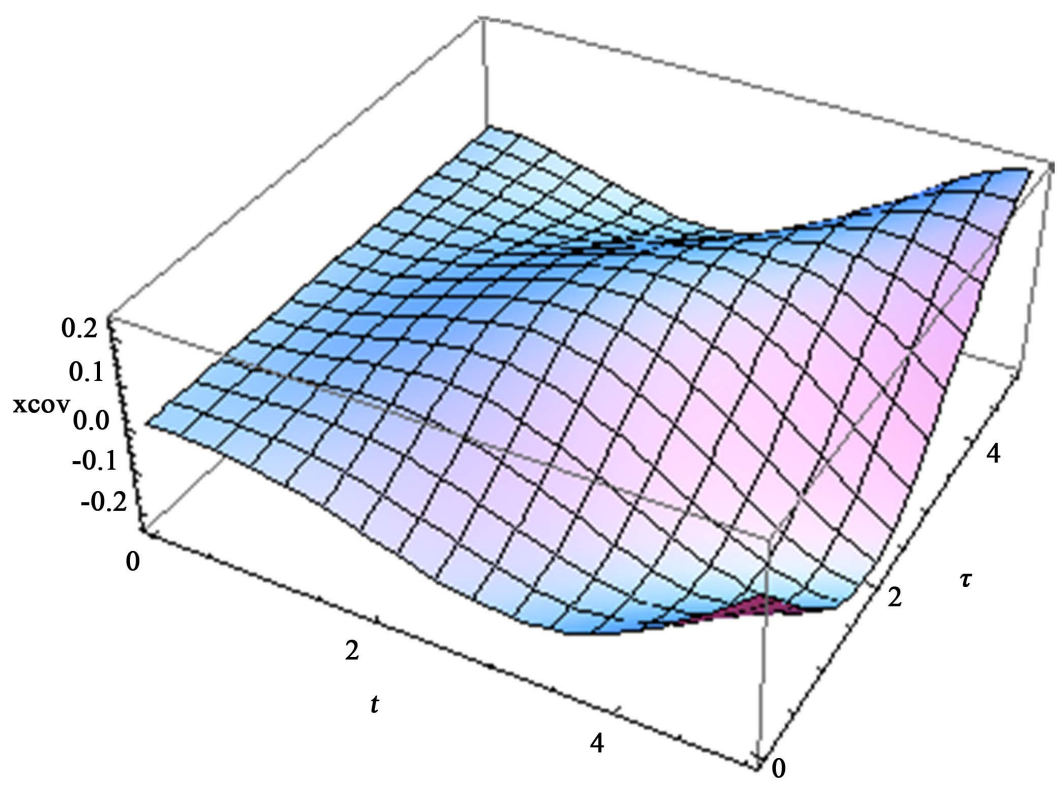

Figure 4. The covariance of $x(t)$ at $\varepsilon=0.3, \omega=1$.

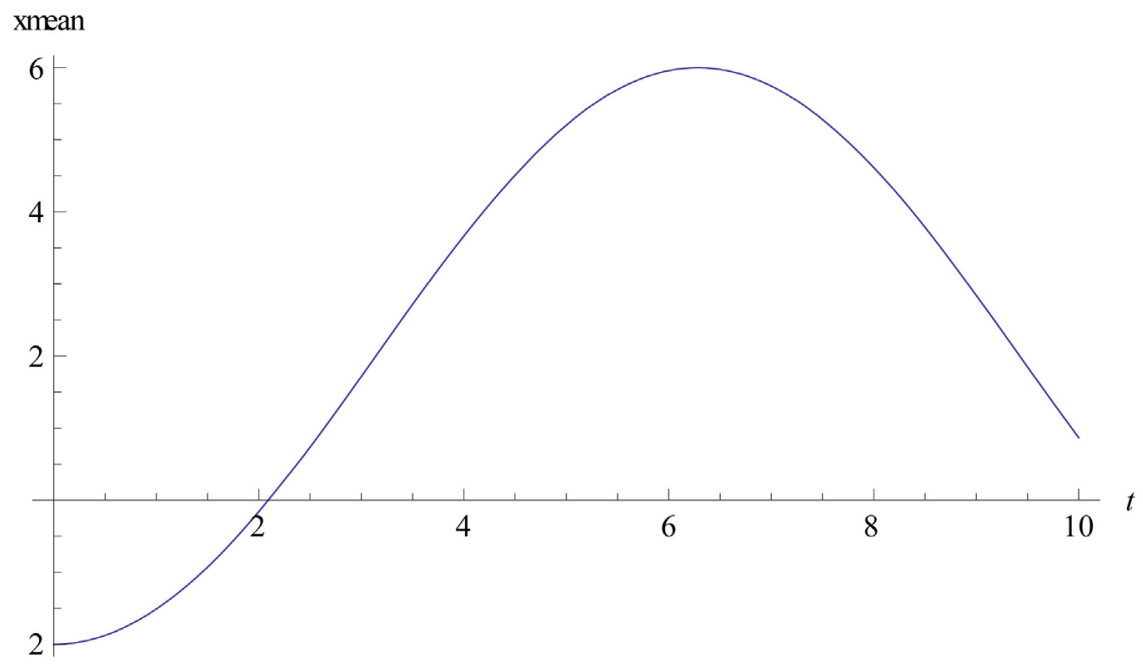

Figure 5. The mean of $x(t)$ at $\omega=0.5$.

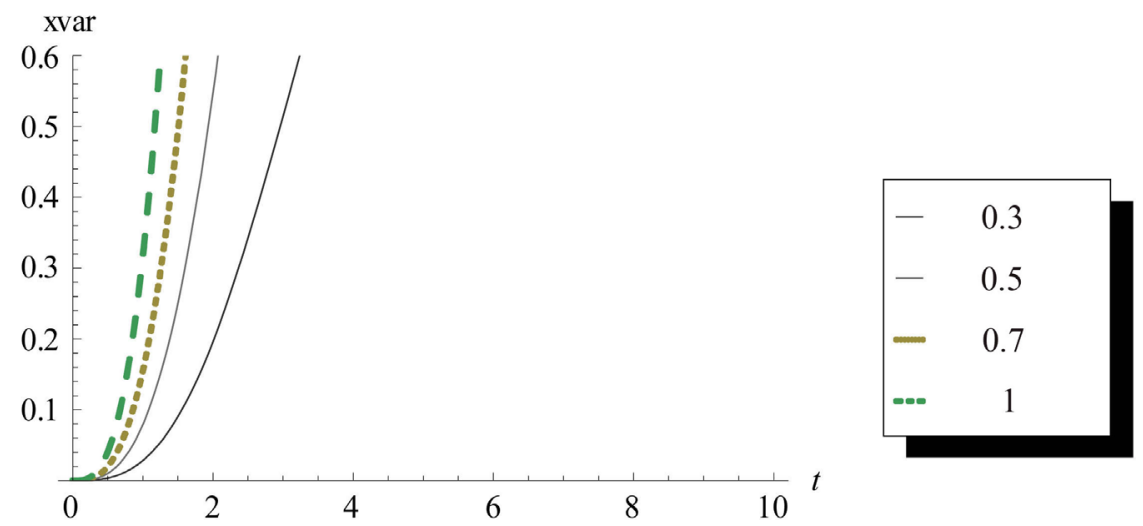

Figure 6. The variance of $x(t)$ at $\omega=0.5$. 


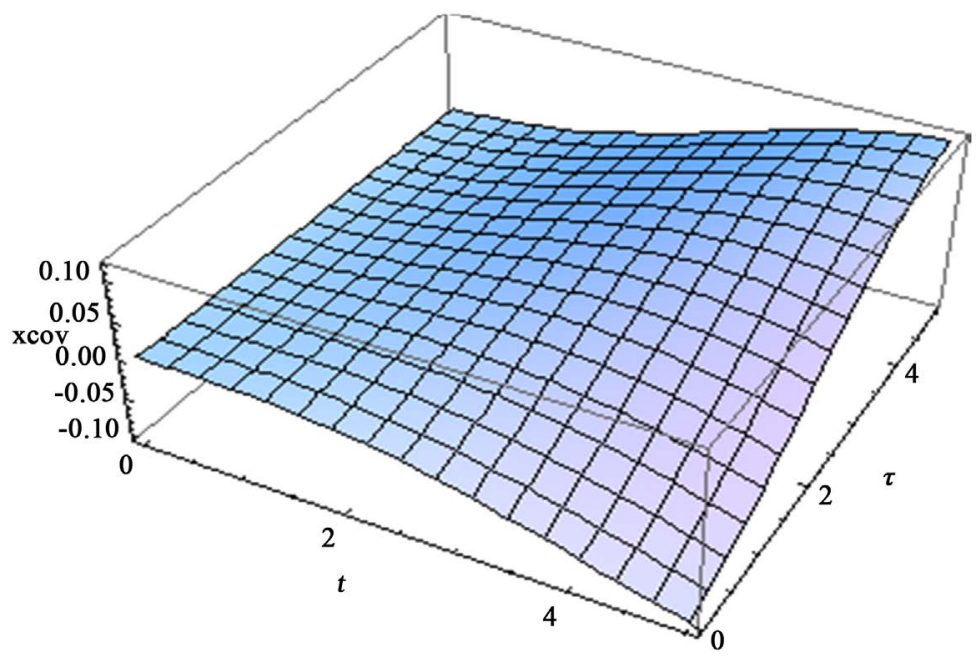

Figure 7. The covariance of $x(t)$ at $\varepsilon=0.1, \omega=0.5$.

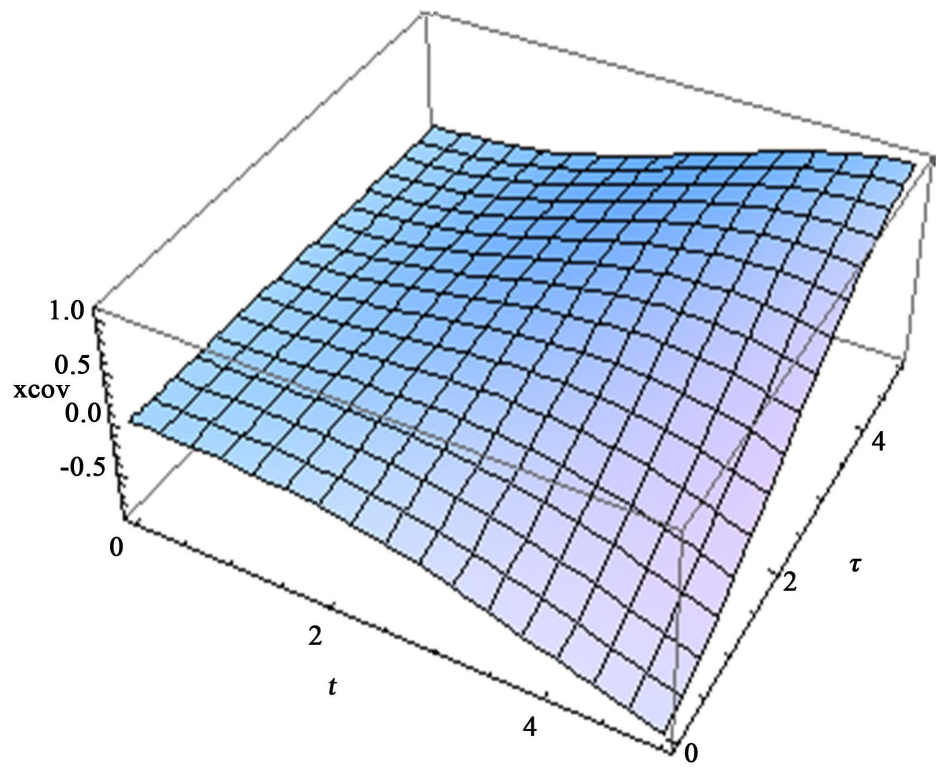

Figure 8. The covariance of $x(t)$ at $\varepsilon=0.3, \omega=0.5$.

$$
\begin{aligned}
x(t)= & x_{0} \cos w t+\frac{\dot{x}_{0}}{\omega} \sin w t+\frac{1}{w}\left[w \int_{0}^{t}(t-u) F(u ; q) \mathrm{d} u\right. \\
& -w^{3} \int_{0}^{t} \frac{(t-u)^{3}}{3 !} F(u ; q) \mathrm{d} u+w^{5} \int_{0}^{t} \frac{(t-u)^{5}}{5 !} F(u ; q) \mathrm{d} u \\
& \left.-w^{7} \int_{0}^{t} \frac{(t-u)^{7}}{7 !} F(u ; q) \mathrm{d} u+\cdots\right] \\
= & x_{0} \cos w t+\frac{\dot{x}_{0}}{\omega} \sin w t+\frac{1}{w} \int_{0}^{t}\left[w(t-u)-\frac{[w(t-u)]^{3}}{3 !}+\cdots\right] F(u ; q) \mathrm{d} u \\
= & x_{0} \cos w t+\frac{\dot{x}_{0}}{w} \sin w t+\frac{1}{w} \int_{0}^{t} \sin w(t-u) F(u ; q) \mathrm{d} u
\end{aligned}
$$


Example:

Let us consider

$$
F(t ; \omega)=e(t)[1+\varepsilon n(t ; q)]
$$

in the previous case-study. By using the decomposition method, the following results are obtained (Figures 1-8).

\section{References}

[1] Rubinstein, R. and Choudhari, M. (2005) Uncertainty Quantification for Systems with Random Initial Conditions Using Wiener-Hermite Expansions. Studies in Applied Mathematics, 114, 167-188. https://doi.org/10.1111/j.0022-2526.2005.01543.x

[2] He J.H. (1999) Homotopy Perturbation Technique. Computer Methods in Applied Mechanics and Engineering, 178, 257-292. https://doi.org/10.1016/S0045-7825(99)00018-3

[3] Nayfeh, A. (1993) Problems in Perturbation. Wiley, New York.

[4] Tamura, Y. and Nakayama, J. (2005) Enhanced Scattering from a Thin Film with One-Dimensional Disorder. Waves in Random and Complex Media, 15, 269-295. https://doi.org/10.1080/17455030500053336

[5] Jahedi, A. and Ahmadi, G. (1983) Application of Wiener-Hermite Expansion to Non-Stationary Random Vibration of a Duffing Oscillator. Journal of Applied Mechanics, Transactions ASME, 50, 436-442. https://doi.org/10.1115/1.3167056

[6] Orabi and Ismail, I. (1988) Response of the Duffing Oscillator to a Non-Gaussian Random Excitation. Journal of Applied Mechanics, Transaction of ASME, 55, 740-743. https://doi.org/10.1115/1.3125861

[7] Kayanuma, Y. and Noba, K. (2001) Wiener-Hermite Expansion Formalism for the Stochastic Model of a Driven Quantum System. Chemical Physics, 268, 177-188. https://doi.org/10.1016/S0301-0104(01)00305-6

[8] Kenny, O. and Nelson, D. (1997) Time-Frequency Methods for Enhancing Speech Proceedings of SPIE-The International Society for Optical Engineering, 3162, 48-57.

[9] De Feriet, K. (1955) Random Solutions of Partial Differential Equations. 3rd Berkeley Symposium on Mathematical Statistics and Probability, Vol. III, 199-208.

[10] El Tawil, M. (1990) On Stochastic Partial Differential Equations. AMSE Review, 14, $1-8$.

[11] El-Tawil, M. and Saleh, M. (1998) The Stochastic Diffusion Equation with a Random Diffusion Coefficient. Ain Shams Engineering Journal, 33, 605-613.

[12] Mckean (1969) Stochastic Integrals. Academic Press, New York.

[13] Kloeden, P.E. and Platen, E. (1992) Numerical Solution of Stochastic Differential Equations. Springer-Verlag, Berlin. https://doi.org/10.1007/978-3-662-12616-5

[14] Arnold, L. (1974) Stochastic Differential Equation Theory and Applications. John Wiley, New York.

[15] Adomian, G. (1988) A Review of the Decomposition in Applied Mathematics. Mathematical Analysis and Applications, 135, 501-544. https://doi.org/10.1016/0022-247X(88)90170-9

[16] Adomian, G. (1992) A Review of the Decomposition Method and Some Recent Results for Nonlinear Equations. Computers \& Mathematics with Applications, 21, 101-127. 
[17] Ahmed, A. (2008) Adomian Decomposition Method: Convergence Analysis and Numerical Approximations. Msc, McMaster University, Hamilton.

[18] Johan, G.J. and Snell, I. (1976) Finite Markov Chains. Springer-Verlag, New York.

[19] Harold, J. (1977) Probability Methods for Approximation in Stochastic Control for Elliptic Equation. Academic Press, New York.

[20] Papoulis, A. (1984) Probability, Random Variables, and Stochastic Processes. McGraw-Hill, New York.

[21] Robert, B. and Melvin, F. (1975) Topics in Stochastic Processes. Academic Press, New York.

[22] Yong, Y. (1995) Convergence of Adomian Method and a Lgorithm for Adomian Polynomials. Journal of Mathematical Analysis and Applications, 33, 442-449.

[23] El-Tawil, M., et al. (2002) Decomposition Solution of Stochastic Nonlinear Oscillator. International Journal of Differential Equations, 6, 441-422.

[24] El-Tawil, M. and Al-Jihany, A. (2009) Approximate Solution of Mixed Nonlinear Stochastic Oscillator. Computers \& Mathematics with Applications, 58, 2236-2259. https://doi.org/10.1016/j.camwa.2009.03.057

[25] Al-Jihany, A. (2010) Comparisons between WHEP and Homotopy Perturbation Techniques in Solving Stochastic Cubic Oscillatory Problems. Computers \& Mathematics with Applications, 58, 19-25. 